\title{
A GeNERALIZATION OF THE DOG BONE SPACE TO $E^{n}$
}

\author{
W. T. EATON ${ }^{1}$
}

\begin{abstract}
In this paper we construct an upper semicontinuous decomposition of $E^{n}(n \geqq 3)$ into points and tame arcs such that the associated decomposition space is distinct from $E^{n}$.
\end{abstract}

The purpose of this paper is to construct an upper semicontinuous decomposition $G$ of $E^{n}, n \geqq 3$, into points and tame arcs such that the decomposition space $E^{n} / G$ is topologically distinct from $E^{n}$. For $n=3$, the example is a modification of R. H. Bing's dog bone space [2]. Unlike the dog bone space, it is not difficult to distinguish our decomposition spaces from Euclidean space.

The main idea for the construction of these decomposition spaces was communicated to us by R. D. Anderson during his visit to the University of Texas in March, 1972. Anderson's idea was roughly the following: Take two disjoint wild Cantor sets in $E^{n}$. To each point in one Cantor set correspond a unique point in the other Cantor set and join the two points with an arc that is locally polygonal modulo its end points. The collection of arcs thus obtained can be constructed so that its union is homeomorphic to the product of a Cantor set and an arc. Such arcs for $n \geqq 4$ are tame and, hopefully, if the Cantor sets are wild enough and the pairings are chosen cleverly, the resulting upper semicontinuous decomposition of $E^{n}$ will have a decomposition space distinct from $E^{n}$.

The difficulty of proving that such decomposition spaces are not $E^{n}$ lies in finding a topological property of $E^{n}$ not shared by the decomposition spaces. We use the following elementary property of $E^{n}$.

THEOREM 1. If $C$ is a Cantor set in $E^{n}(n \geqq 3), U$ is an open set containing $C$, and $f$ and $g$ are maps from a 2-cell $D$ into $E^{n}$, then there exist maps $f^{\prime}$ and $g^{\prime}$ from $D$ into $E^{n}$ such that $f^{\prime}\left|f^{-1}\left(E^{n}-U\right)=f\right| f^{-1}\left(E^{n}-U\right)$, $g^{\prime}\left|g^{-1}\left(E^{n}-U\right)=g\right| g^{-1}\left(E^{n}-U\right), \cdot f^{\prime}\left(f^{-1}(U)\right) \subset U, \quad g^{\prime}\left(g^{-1}(U)\right) \subset U \quad$ and $f^{\prime}(D) \cap g^{\prime}(D) \cap C=\varnothing$.

Received by the editors July 24,1972 .

AMS (MOS) subject classifications (1970). Primary 57A15, 57A10, 54A30; Secondary 57A35, 57A45.

Key words and phrases. Tame arcs, wild embeddings, cellular maps, upper semicontinuous.

${ }^{1}$ This work was supported in part by NSF Grant GP-29400X.

(c) American Mathematical Society 1973 
Proof. By adjusting $f \mid f^{-1}(U)$ and $g \mid g^{-1}(U)$ if necessary, we may assume that (1) $f$ and $g$ are locally PL modulo $f^{-1}\left(E^{g}-U\right)$ and $g^{-1}\left(E^{g}-U\right)$ respectively, and (2) $f(D) \cap U$ and $g(D) \cap U$ are in general position. For $n \geqq 5, f(D) \cap g(D) \cap U=\varnothing$ so we may take $f^{\prime}=f$ and $g^{\prime}=g$. In the other cases we obtain the required maps $f^{\prime}$ and $g^{\prime}$ by composing $f$ and $g$, respectively, with a space homeomorphism that pushes the tame one $(n=3)$ or zero $(n=4)$ dimensional set $f(D) \cap g(D) \cap U$ off $C$.

Wild Cantor sets in $E^{n}$. The Cantor sets we use are similar to the generalizations of Antoine's necklace [1] discussed by W. A. Blankinship [4] in 1950.

A wild Cantor set in $E^{n}(n \geqq 3)$ may be constructed as the intersection of a nest $M_{1} \supset$ Int $M_{1} \supset M_{2} \supset$ Int $M_{2} \supset M_{3} \cdots$ of PL $n$-manifolds $M_{j}$ with boundary such that each component of each $M_{j}$ is homeomorphic to the solid $n$-torus $B^{2} \times S_{1}^{1} \times S_{2}^{1} \times \cdots \times S_{n-2}^{1}$ where $B^{2}$ is a 2-cell and $S_{i}^{1}$ is a 1 -sphere. If $A$ is a component of $M_{j}$ then the components $A_{1}, \cdots, A_{k}$ of $M_{j+1}$ in $A$ are embedded in $A$ in the following canonical way.

Let $P_{i}(i=1, \cdots, n-2)$ be the natural projection of the solid $n$-torus $A \sim B^{2} \times S_{1}^{1} \times \cdots \times S_{i}^{1} \times \cdots \times S_{n-2}^{1}$ onto its factor space $B^{2} \times S_{i}^{1}$. For each integer $i(i=1, \cdots, n-2)$ we associate an embedding of $k$ solid $n$-tori $A_{1}, \cdots, A_{k}$ in $A$. Since there exists a homeomorphism of $A$ onto itself which interchanges $S^{1}$ factors, the $n-2$ embeddings are actually alike topologically. Let $i$ be fixed. The factor space $B^{2} \times S_{i}^{1}$ is the large solid

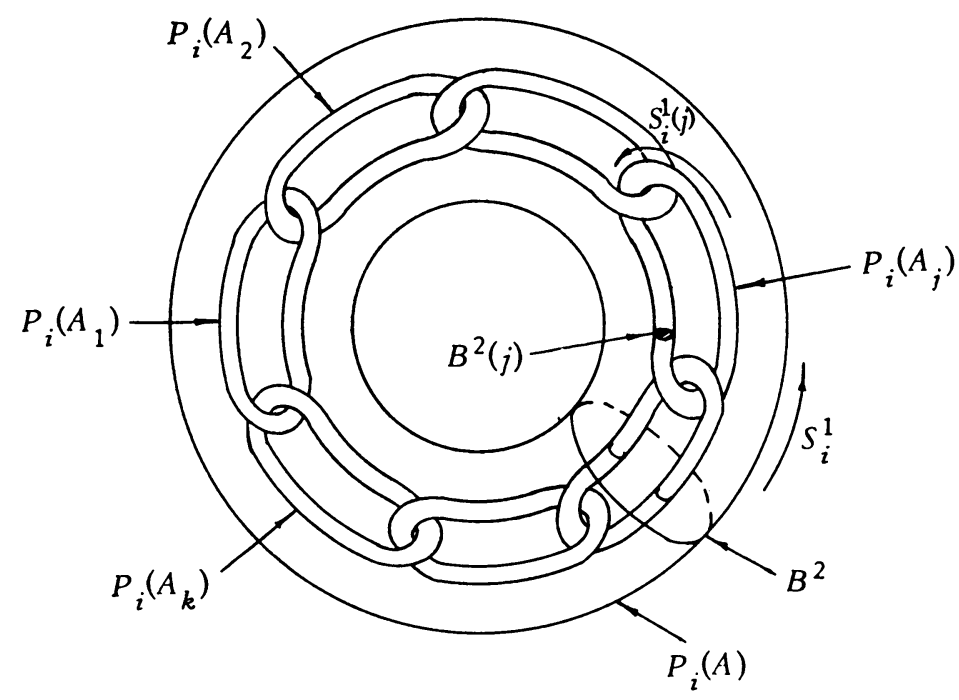

Figure 1 
3-torus in Figure 1, and the embeddings of the projections $P_{i}\left(A_{1}\right), \cdots$, $P_{i}\left(A_{k}\right)$ of the solid $n$-tori $A_{1}, \cdots, A_{k}$ in $P_{i}(A) \sim B^{2} \times S_{i}^{1}$ are the smaller solid 3-tori linked one to another around the factor $S_{i}^{1}$ as in Figure 1. The solid $n$-torus $A_{j}$ is like $A$ in $n-3$ of its factors; in fact, if $B^{2}(j) \times S^{1}(j)$ is the solid 3-torus in Figure 1, then $A_{j}=B^{2}(j) \times S_{1}^{1} \times \cdots \times S_{i-1}^{1} \times$ $S_{i}^{1}(j) \times S_{i+1}^{1} \times \cdots \times S_{n-2}^{1}$. The number $k$ of $A_{j}$ 's may be arbitrarily large but always at least 3 . The embedding of the solid $n$-tori $\left\{A_{j}\right\}$ in the solid $n$-torus $A$ will be referred to as an Antoine embedding.

We start the construction of a wild Cantor set by taking $M_{1}$ to be any solid $n$-torus in $E^{n}$. We would like to make the diameter of the components of $M_{2}$ in $M_{1}$ small relative to the diameter of $M_{1}$ by using an Antoine embedding of solid $n$-tori in $M_{1}=A$. However, no matter how large the integer $k$ we can make a component $A_{j}$ of $M_{2}$ small only in two of its coordinates, namely $B^{2}(j)$ and $S^{1}(j)$ of the previous paragraph. Thus we may make all the components small at the second stage only if $n=3$. The remedy for the difficulty for $n>3$ is to use $n-2$ stages to make components small relative to the diameter of $M_{1}$. That is, link the solid $n$-tori of $M_{2}$ about the first $S^{1}$ factor of $M_{1}$ (use $P_{1}$ of the previous paragraph), then in each component of $M_{2}$ link the solid $n$-tori about the second $S^{1}$ factor, etc. Thus the components of $M_{n-1}$ may be made of arbitrarily small diameter, and by repeating the process we can insure that $\bigcap M_{i}$ is a Cantor set.

To show that the Cantor set $M=\bigcap M_{i}$ is wild, Blankinship [4] computed explicitly $\pi_{1}\left(E^{n}-M\right)$ and showed that the group was nontrivial by representing it nontrivially in the symmetric group $S_{6}$. We are more interested in geometry than its amusing derivatives; consequently, we proceed alternatively by using the following definition and lemma.

Definition 1. Let $A$ be a solid $n$-torus $B^{2} \times S_{1}^{1} \times \cdots \times S_{n-2}^{1}$ in $E^{n}$. A PL map $f$ from a 2-cell $D$ into $E^{n}$ is regular with respect to $A$ if

(1.1) $f(D)$ and $\mathrm{Bd} A$ are in general position,

(1.2) $f \mid \mathrm{Bd} D$ is a nontrivial loop in $\mathrm{Bd} A$,

(1.3) there exists a collar $C$ of $\mathrm{Bd} D$ in $D$ such that $f$ (Int $C) \subset$ Int $A$, and

(1.4) if $K$ is a component of $f^{-1}(f(\operatorname{Int} D) \cap \mathrm{Bd} A)(K$ is necessarily a simple closed curve by (1.1)) then $f \mid K$ is a trivial loop in $\operatorname{Bd} A$.

LeMmA 1. If $A$ is a solid $n$-torus $(n \geqq 3) B^{2} \times S_{1}^{1} \times \cdots \times S_{n-2}^{1}$ in $E^{n}$, the solid $n$-tori $A_{1}, \cdots, A_{k}$ are Antoine embedded in $A, f$ is a map from $a$ 2-cell $D$ into $E^{n}$ such that $f$ is regular with respect to $A$ and $f(D)$ and $\cup \mathrm{Bd} A_{i}$ are in general position, then there exist an integer $j$ and a subdisk $D^{*}$ of $D$ such that $f \mid D^{*}$ is regular with respect to $A_{j}$.

Proof. Since $A_{1}, \cdots, A_{k}$ are Antoine embedded in $A$, for some $i$ the natural projection map $P_{i}$ of $A \sim B^{2} \times S_{1}^{1} \times \cdots \times S_{i}^{1} \times \cdots \times S_{n-2}^{1}$ onto 
its factor space $B^{2} \times S_{i}^{1}$ takes the $A_{j}$ 's to the solid 3-tori linked around $S_{i}^{1}$ as indicated in Figure 1. We first show that there exists a component $K$ of $f^{-1}\left(\cup \operatorname{Bd} A_{j}\right)$ and an integer $j$ such that $f \mid K$ is nontrivial in $\operatorname{Bd} A_{j}$. We assume the contrary and let $a_{0}, a_{1}, \cdots, a_{n-2}$ be a free basis for the free abelian group $\pi_{1}(\operatorname{Bd} A)$. We may take $a_{0}=\left[\operatorname{Bd} B^{2}\right], a_{1}=\left[S_{1}^{1}\right], \cdots$, $a_{n-2}=\left[S_{n-2}^{1}\right]$ so that $a_{1}, \cdots, a_{n-2}$ is a free basis for the free abelian group $\pi_{1}(A)$. By (1.3), (1.4) and our supposition, we may adjust $f$ by cutting off trivial loops on $\left(\cup \mathrm{Bd} A_{j}\right) \cup \mathrm{Bd} A$ to obtain a map $f^{\prime}$ from $D$ into $A$ such that $f^{\prime}|\operatorname{Bd} D=f| \operatorname{Bd} D$ and $f^{\prime}(D) \cap\left(\bigcup A_{j}\right)=\varnothing$. Thus $f \mid \operatorname{Bd} D$ is a nontrivial loop in $\mathrm{Bd} A$ (1.2) and trivial in $A$ and, consequently, has the reduced form $a_{0}^{r}$ for some nonzero integer $r$. It follows that $P_{i} f^{\prime} \mid \operatorname{Bd} D$ is nontrivial in the boundary of the solid 3-torus $B^{2} \times S_{i}^{1}$ and $P_{i} f^{\prime}(D)$ misses each of the solid 3-tori $P_{i}\left(A_{j}\right)$. These facts contradict that the $P_{i}\left(A_{j}\right)$ 's link one to another around the $S_{i}^{1}$ factor of $B^{2} \times S_{i}^{1}$ (see [3, Theorems 9 and 11] for the techniques to prove this 3-space result). Thus there are components $K$ of $f^{-1}\left(\bigcup \mathrm{Bd} A_{j}\right)$ such that $f \mid K$ is nontrivial in $\bigcup \operatorname{Bd} A_{j}$.

Let $K$ be an innermost such component on $D$, let $D^{*}$ be the subdisk in $D$ that $K$ bounds and let $j$ be the integer such that $f(K) \subset \mathrm{Bd} A_{j}$. We complete the proof by showing that $f \mid D^{*}$ is regular with respect to $A_{j}$. Note that it is only necessary to show that there exists a collar $C^{*}$ of $D^{*}$ such that $f\left(\operatorname{Int} C^{*}\right) \subset \operatorname{Int} A_{j}$. We suppose the contrary; that is, suppose there exists a collar $C^{*}$ of $D^{*}$ such that $f\left(\operatorname{Int} C^{*}\right) \subset\left(\operatorname{Ext} A_{j}\right) \cap(\operatorname{Int} A)$. Since each component $J \neq \mathrm{Bd} D^{*}$ of $\left(f \mid D^{*}\right)^{-1}\left(\left(\cup \operatorname{Bd} A_{m}\right) \cup \operatorname{Bd} A\right)$ has the property that $f \mid J$ is trivial on $\left(\cup \operatorname{Bd} A_{m}\right) \cup \operatorname{Bd} A$, we may adjust the map $f \mid D^{*}$ to obtain a map $f^{\prime}$ such that $f^{\prime}\left(\right.$ Int $\left.D^{*}\right) \subset($ Int $A)-\cup A_{m}$ and $f^{\prime}\left|\operatorname{Bd} D^{*}=f\right| \operatorname{Bd} D$. The loop $f \mid \operatorname{Bd} D$ is thus trivial in $A$ and, consequently, cannot contain the factors $a_{1}, \cdots, a_{i-1}, a_{i+1}, \cdots, a_{n-1}$ in its reduced form. Thus the reduced form of $f \mid \mathrm{Bd} D^{*}$ contains only the factors $a_{0}(j)=\left[\mathrm{Bd} B^{2}(j)\right]$ and $a_{i}(j)=\left[S_{i}^{1}(j)\right]$ in $\pi_{1}\left(\mathrm{Bd} A_{j}\right)$ (see Figure 1), and since $f \mid \mathrm{Bd} D^{*}$ is nontrivial on $\mathrm{Bd} A_{j}$, the reduced form of $f \mid \mathrm{Bd} D^{*}$ contains at least one of $a_{0}(j)$ and $a_{i}(j)$ as factors. It follows that $P_{i} f^{\prime} \mid \operatorname{Bd} D^{*}$ is nontrivial on $\mathrm{Bd} P_{i}\left(A_{j}\right)$ and $P_{i} f^{\prime}\left(\operatorname{Int} D^{*}\right) \cap\left(\bigcup_{m} P_{i}\left(A_{m}\right)\right)=\varnothing$. Since $P_{i} f^{\prime}$ (Int $\left.D^{*}\right) \cap P_{i}\left(A_{j}\right)=\varnothing$ we have that $P_{i} f^{\prime} \mid \mathrm{Bd} D^{*}$ contains the factor $a_{i}(j)$, but this is impossible since some $P_{i}\left(A_{m}\right)$ links $P_{i}\left(A_{j}\right)$ denying that $f^{\prime}\left(\right.$ Int $\left.D^{*}\right) \cap P_{i}\left(A_{m}\right)$ is empty. Hence $D^{*}$ contains a collar $C^{*}$ such that $f\left(\right.$ Int $\left.C^{*}\right) \subset \operatorname{Int} A_{j}$ and $f \mid D^{*}$ is regular with respect to $A_{j}$.

We will use Lemma 1 and Theorem 1 to establish that our decomposition spaces must fail to be $E^{n}$. We first use Lemma 1 to show that the Cantor set $\bigcap M_{j}$ is wild in $E^{n}$.

Suppose $\cap M_{j}$ is not wild in $E^{n}$; then with a slight adjustment of $B^{2}$ in $M_{1}$ we may assume that $B^{2}$ misses $\bigcap M_{j}$. We realize the adjusted $B^{2}$ as 
the image of a disk $D$ under a PL map $f$. We may assume that $f(D)$ is in general position with respect to each $\mathrm{Bd} M_{j}$. Lemma 1 is used repeatedly to obtain a sequence $D=D_{1} \supset D_{2} \supset D_{3} \supset \cdots$ of subdisks of $D$ and a sequence $A_{i_{1}} \supset A_{i_{1} i_{2}} \supset A_{i_{1} i_{2} i_{3}} \supset \cdots$ of solid $n$-tori such that $f \mid D_{j}$ is regular with respect to the solid $n$-torus $A_{i_{1} \cdots i_{i}}$, where $A_{i_{1} \cdots i_{j}}$ is a component of $M_{j}$. It follows that $f\left(\bigcap_{j} D_{j}\right)=\bigcap A_{i_{1} \cdots i_{j}}$ which is a point of the Cantor set $\bigcap M_{j}$. Thus a contradiction is reached and we have that $\bigcap M_{j}$ must be wild.

The construction. The Cantor sets we use are very much like the Antoine-Blankinship type. We realize each Cantor set as the intersection of a nest $M_{1} \supset$ Int $M_{1} \supset M_{2} \supset$ Int $M_{2} \supset \cdots$ of PL $n$-manifolds with boundary in $E^{n}$ such that each component of each $M_{j}$ is a solid $n$-torus $B^{2} \times$ $S_{1}^{1} \times \cdots \times S_{n-2}^{1}$. If $A \sim B^{2} \times S_{1}^{1} \times \cdots \times S_{n-2}^{1}$ is a component of $M_{j}$ then there are $k^{2}$ components $A_{11}, \cdots, A_{k k}$ of $M_{j+1}$ embedded in $A$ so that for some $i$ the natural projection map $P_{i}$ of $A$ onto its factor space $B^{2} \times S_{i}^{1}$ carries the solid $n$-tori $A_{11}, \cdots, A_{k k}$ onto the solid 3 -tori $P_{i}\left(A_{11}\right), \cdots$, $P_{i}\left(A_{k k}\right)$ embedded in $P_{i}(A) \sim B^{2} \times S_{i}^{1}$ as in Figure 2. As Figure 2 illustrates, for each $j$ the solid $n$-tori $A_{j 1}, \cdots, A_{j k}$ are very close approximations to each other; in fact, if for each $j$ we choose $i_{j} \in\{1, \cdots, k\}$ then $A_{1 i_{1}}, \cdots$, $A_{k i_{k}}$ are Antoine embedded in $A$. The components of $M_{k}$ are made small as $k \rightarrow \infty$ in the same way as for the Antoine-Blankinship Cantor sets; that is, the $S^{1}$ factors of the solid $n$-torus $A$ that the $A_{i j}$ link around alternate as $k \rightarrow \infty$.

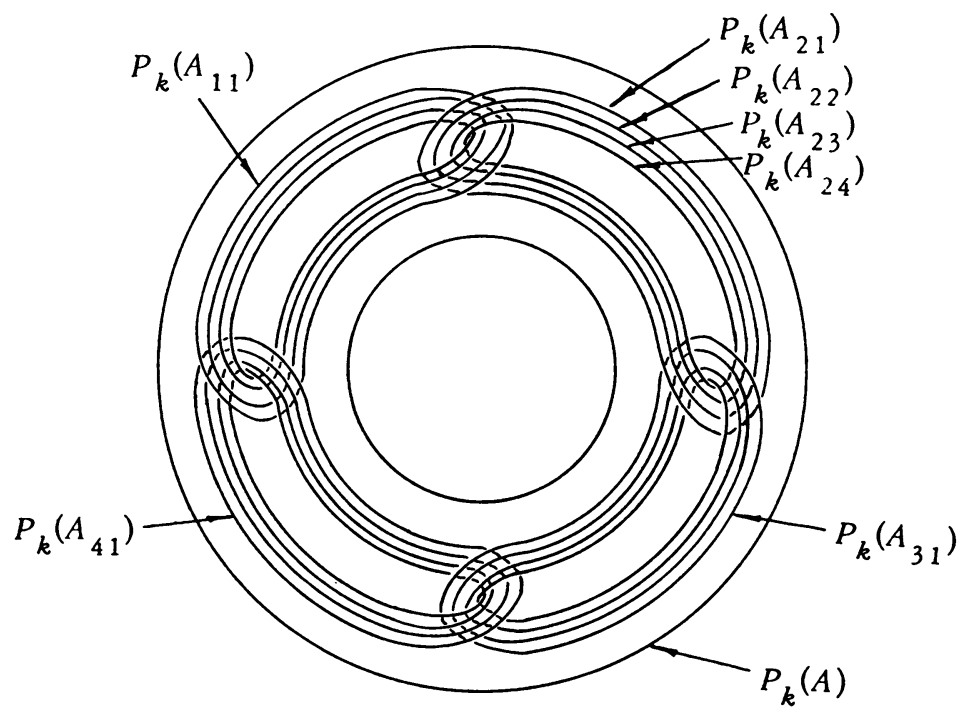

FIGURE 2 


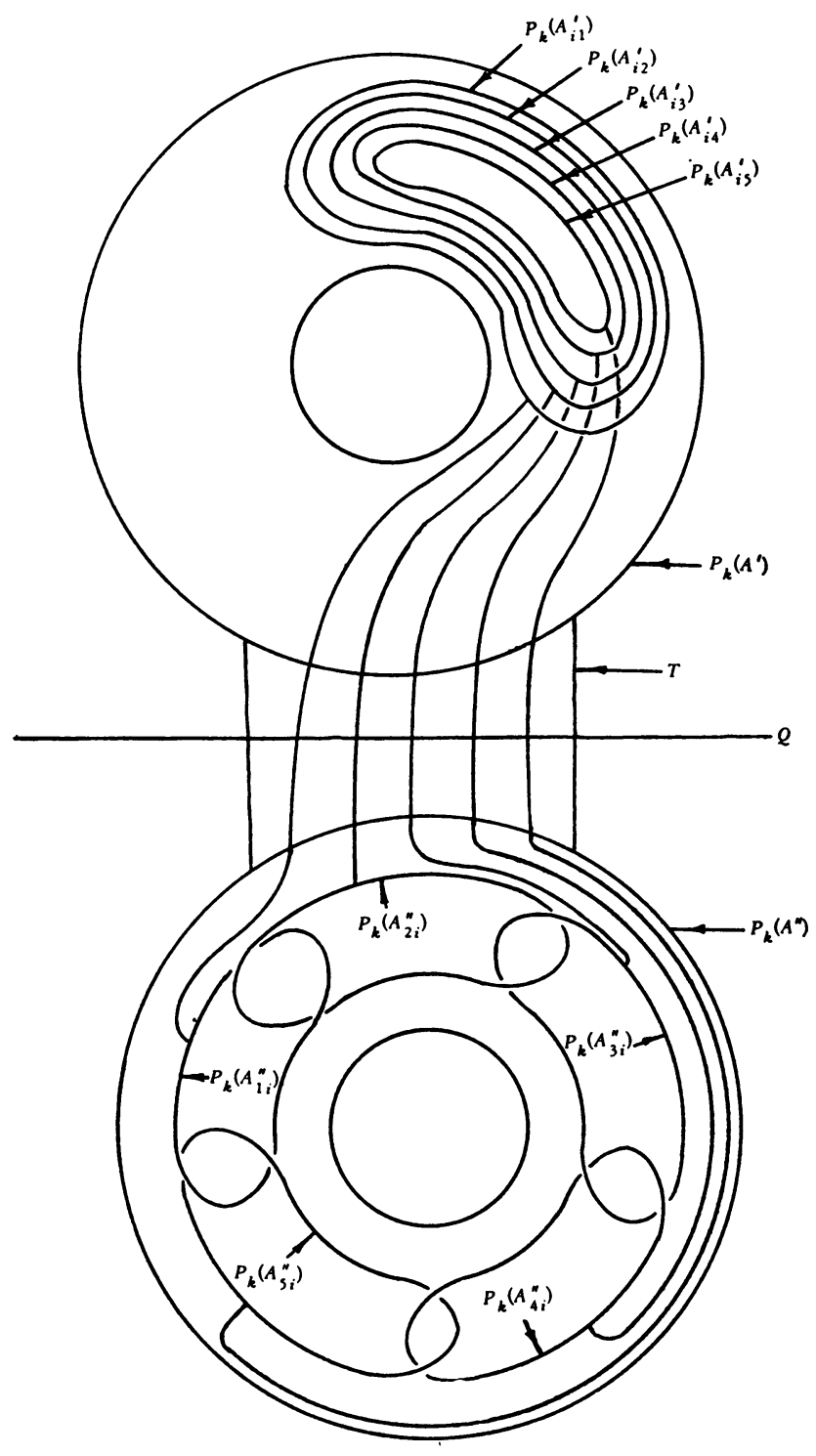

Figure 3 
For convenience we locate our disjoint Cantor sets on opposite sides of an (n-1)-dimensional hyperplane $Q$ in $E^{n}$. We denote the manifolds used to describe the Cantor set above $Q$ with primed letters and the manifolds used to describe the Cantor set below $Q$ by double primed letters. The upper semicontinuous decomposition $G$ of $E^{n}$ into points and tame arcs is now easy to describe.

The $n$-manifolds $M_{1}^{\prime}$ and $M_{1}^{\prime \prime}$ are solid $n$-tori; that is, $M_{1}^{\prime}$ and $M_{1}^{\prime \prime}$ each have one component homeomorphic to $B^{2} \times S_{1}^{1} \times \cdots \times S_{n-2}^{1}$. We connect $M_{1}^{\prime}$ and $M_{1}^{\prime \prime}$ with an $n$-tube, $T_{1}$, and we denote the union of $M_{1}^{\prime}, M_{1}^{\prime \prime}$, and the tube $T_{1}$ by $M_{1}$. Each component $A_{i j}^{\prime}$ of $M_{2}^{\prime}$ is connected to a unique component $A_{j i}^{\prime \prime}$ of $M_{2}^{\prime \prime}$ by an $n$-tube $T_{i j}$ in Int $M_{1}$ that runs straight through the tube $T_{1}$ (see Figure 3). We denote the union of $A_{i j}^{\prime}, A_{j i}^{\prime \prime}$, and $T_{i j}$ by $A_{i j}$. The $T_{i j}$ 's are constructed disjointly and the union of the $k^{2} n$-manifolds $A_{i j}$ is $M_{2}$. Inductively, if $A=A^{\prime} \cup A^{\prime \prime} \cup T$ is a component of $M_{k}$ where $A^{\prime}, A^{\prime \prime}$ are solid $n$-tori and $T$ is an $n$-tube connecting them, then the components, $\left\{A_{i j}\right\}$, of $M_{k+1}$ in $A$ are obtained by connecting each of the $k^{2}$ solid $n$-tori $A_{i j}^{\prime}$ in $A^{\prime}$ to a unique solid $n$-torus $A_{j i}^{\prime \prime}$ in $A^{\prime \prime}$ by an $n$-tube $T_{i j}$ in Int $A$ that runs straight through the $n$-tube $T$. The $T_{i j}$ 's are constructed disjointly as thin regular neighborhoods of PL arcs. Figure 3 illustrates the iterated step in which the $n$-tubes connecting the $A_{i j}^{\prime}$ 's and $A_{j i}^{\prime \prime}$ 's are schematically pictured as connecting $P_{k}\left(A_{i j}^{\prime}\right)$ 's and $P_{k}\left(A_{j i}^{\prime \prime}\right)$ 's.

The nondegenerate elements of our upper semicontinuous decomposition $G$ of $E^{n}$ are the components of $\bigcap M_{i}$. Since the components of $M_{i}^{\prime}$ and $M_{i}^{\prime \prime}$ become small as $i$ gets large and, in the iterative step, we require that each $n$-tube $T_{i j}$ be thin and run straight through the $n$-tube $T$ of the preceding stage, each nondegenerate element of $G$ is an arc that is locally polygonal modulo its end points. It is well known and an easy exercise to show that such arcs are tame if $n \geqq 4$. For $n=3$, we can insure that the arcs are tame by also requiring that the 3-tubes do not pass through the holes of the solid 3-tori they connect. Thus each arc will be unknotted at its ends.

The following lemma will be used iteratively to help establish that $E^{n} / G$ is not topologically $E^{n}$.

LEMMA 2. If $A=A^{\prime} \cup A^{\prime \prime} \cup T$ is a component of $M_{k}$ from the description of $G$ above, where $A^{\prime}$ and $A^{\prime \prime}$ are solid n-tori and $T$ is an n-tube connecting them, and $f$ and $g$ are $P L$ maps from a 2-cell $D$ into $E^{n}$ such that $f(D) \cup g(D)$ and $\left(\cup \mathrm{Bd} A_{i j}^{\prime}\right) \cup\left(\cup \mathrm{Bd} A_{j i}^{\prime \prime}\right)$ are in general position, $f$ is regular with respect to $A^{\prime}$, and $g$ is regular with respect to $A^{\prime \prime}$, then there exist subdisks $D_{0}$ and $E_{0}$ of $D$ and a component $A_{i j}=A_{i j}^{\prime} \cup T_{i j} \cup A_{j i}^{\prime \prime}$ of $M_{k+1}$ in $A$ such that $f \mid D_{0}$ is regular with respect to $A_{i j}^{\prime}$ and $g \mid E_{0}$ is regular with respect to $A_{j i}^{\prime \prime}$. 
Proof. There exists an integer $i$ such that for all $m$ there exists a subdisk $D_{m}$ of $D$ such that $f \mid D_{m}$ is regular with respect to $A_{i m}^{\prime}$. For suppose the contrary, then for each $i$ we may pick an integer $j_{i}$ such that $f \mid D^{\prime}$ is not regular with respect to $A_{i j_{i}}^{\prime}$ for each subdisk $D^{\prime}$ of $D$. But $A_{1 j_{i}}^{\prime}, \cdots, A_{k j_{k}}^{\prime}$ form an Antoine embedding in $A^{\prime}$, thus we have a contradiction to Lemma 1.

The solid $n$-tori $A_{1 i}^{\prime \prime}, \cdots, A_{k i}^{\prime \prime}$ form an Antoine embedding in $A^{\prime \prime}$. By Lemma 1 , there exist a subdisk $E_{0}$ of $D$ and an integer $j$ such that $g \mid E_{0}$ is regular with respect to $A_{j i}^{\prime \prime}$. The proof is completed by setting $D_{0}=D_{j}$.

THEOREM 2. If $G$ is the upper semicontinuous decomposition described above then $E^{n} / G$ is not topologically $E^{n}(n \geqq 3)$.

Proof. We suppose that $E^{n} / G \sim E^{n}$ and let $P$ be the natural projection map of $E^{n}$ onto $E^{n} / G \sim E^{n}$ associated with the decomposition $G$. Let $f$ and $g$ be homeomorphisms from a disk $D$ onto the $B^{2}$ factors of the solid $n$-tori $M_{1}^{\prime}$ and $M_{1}^{\prime \prime}$, respectively. We may assume that $f(D)$ and $g(D)$ miss the $n$-tube $T_{1}$ and that $f$ and $g$ are regular with respect to $M_{1}^{\prime}$ and $M_{1}^{\prime \prime}$, respectively.

We now use our supposition and Theorem 1 to adjust $f$ and $g$ to obtain maps $f^{\prime \prime}, g^{\prime \prime}$ and an integer such that $f^{\prime \prime}(D)$ and $g^{\prime \prime}(D)$ do not intersect a common component of $M_{j}$ and $f^{\prime \prime}$ and $g^{\prime \prime}$ are regular with respect to $M_{1}^{\prime}$ and $M_{1}^{\prime \prime}$, respectively. By Theorem $1, P f(D)$ and $P g(D)$ may be adjusted in $P\left(\right.$ Int $\left.M_{1}\right)$ to obtain maps $f^{\prime}$ and $g^{\prime}$ from $D$ into $P\left(\right.$ Int $\left.M_{1}\right)$ such that $f^{\prime}|\operatorname{Bd} D=P f| \operatorname{Bd} D, g^{\prime}|\operatorname{Bd} D=P g| \operatorname{Bd} D$, and $f^{\prime}(D) \cap g^{\prime}(D) \cap P\left(\cap M_{i}\right)=$ $\varnothing$. Since $f^{\prime}(D) \cap g^{\prime}(D) \cap P\left(\cap M_{i}\right)=\varnothing$, there exists an integer $j$ such that $f^{\prime}(D)$ and $g^{\prime}(D)$ do not intersect a common component of $P\left(M_{j}\right)$. Since the components of $\cap M_{i}$ are cellular, there exists a collection of $n$-cells $\left\{C_{i}\right\}$ such that $C_{i} \subset$ Int $M_{j}$ and each component of $\cap M_{j}$ lies in the interior of some $C_{i}$. The collection $\left\{\right.$ Int $\left.P\left(C_{i}\right)\right\}$ covers $P\left(\cap M_{i}\right)$, thus there exists an integer $k$ such that each component of $P\left(M_{j+k}\right)$ lies in Int $P\left(C_{i}\right)$ for some $i$. Let $F$ and $G$ be, respectively, the component of $\left(f^{\prime}\right)^{-1}\left(E^{n}-P\left(M_{j+k}\right)\right)$ and the component of $\left(g^{\prime}\right)^{-1}\left(E^{n}-P\left(M_{j+k}\right)\right)$ that contain Bd $D$. Since $\cap M_{i} \subset$ Int $M_{j+k}, P^{-1}$ is a well defined map on $f^{\prime}(F) \cup g^{\prime}(G)$. We obtain the map $f^{\prime \prime}$ by extending $P^{-1} f^{\prime} \mid F$ to $D$ so that $f^{\prime \prime}(K) \subset$ Int $C_{i}$ where $K$ is a component of $D-F$ and $C_{i}$ is an $n$-cell that contains $P^{-1} f^{\prime}(\operatorname{Bd} K)$. The map $g^{\prime \prime}$ is obtained similarly.

The existence of the integer $j$ and the maps $f^{\prime \prime}$ and $g^{\prime \prime}$ is contrary to Lemma 2, however. By applying Lemma 2 repeatedly, we obtain nests $D=F_{1} \supset F_{2} \supset \cdots \supset F_{j}, \quad D=G_{1} \supset G_{2} \supset \cdots \supset G_{j}$ of subdisks of $D$, and nests $A_{1} \supset A_{2} \supset A_{3} \supset \cdots \supset A_{j}$ such that $A_{i}$ is a component of $M_{i}$ and

$$
f^{\prime \prime}\left(F_{i}\right) \cap A_{i} \neq \varnothing \text { and } g^{\prime \prime}\left(G_{i}\right) \cap A_{i} \neq \varnothing \quad \text { for } i=1, \cdots, j \text {. }
$$

Thus $f^{\prime \prime}(D)$ and $g^{\prime \prime}(D)$ intersect a common component of $M_{j}$. 
Assuming that $E^{n} / G \sim E^{n}$ leads to a contradiction, thus $E^{n} / G$ is not topologically $E^{n}$.

\section{REFERENCES}

1. M. L. Antoine, Sur l'homeomorphie de deux figures et de leurs voisinages, J. Math. Pures Appl. 86 (1921), 221-325.

2. R. H. Bing, $A$ decomposition of $E^{3}$ into points and tame arcs such that the decomposition space is topologically different from $E^{3}$, Ann. of Math. (2) 65 (1957), 484-500. MR 19, 1187.

3. - A wild surface each of whose arcs is tame, Duke Math. J. 28 (1961), 1-15. MR 23 \#A630.

4. W. A. Blankinship, Generalization of a construction of Antoine, Ann. of Math. (2) 53 (1951), 276-297. MR 12, 730.

Department of Mathematics, University of Texas, Austin, Texas 78712 